\title{
On-farm use of disease alerts generated by precision dairy technology
}

\author{
E. A. Eckelkamp ${ }^{1 *}\left(\right.$ ) and J. M. Bewley ${ }^{2}$ () \\ ${ }^{1}$ Animal Science Department, Institute of Agriculture, University of Tennessee, Knoxville 37996 \\ ${ }^{2}$ Alltech, Nicholasville, KY 40356
}

\begin{abstract}
Wearable precision dairy monitoring (PDM) technologies currently used to detect estrus may provide insight into disease detection. However, the incorporation of PDM into farm management and its perceived usefulness for dairy producers have not been explored. As the targeted end users of these products, information is needed on how producers use generated disease alerts as well as barriers to adoption and usefulness. The objective of this research was to assess the perceived usefulness producers attributed to alerts from a daily generated alert list designed to identify sick or injured cows or cows that experienced a major management change. Data from 1,171 cows on 4 commercial farms in Kentucky were collected from October 2015 to October 2016. Each cow was equipped with 2 PDM technologies: a leg tag (measuring activity in steps/d and lying time in $\mathrm{h} / \mathrm{d}$ ) and a neck collar (measuring eating time in $\mathrm{h} / \mathrm{d}$ ). Alerts were generated based on an individual cow's decrease of $\geq 30 \%$ in activity, lying, and eating time compared with each cow's 10-d moving mean. Producers sorted alerts into 3 overall categories: (1) the cow alert was perceived to be true and the cow was visually checked, (2) the cow alert was perceived to be true, but the cow was not visually checked, and (3) the cow alert behavior change was doubted by the producer and the cow was not visually checked. Further subdivisions were also provided to explain the choice for an overall category. Over the year, 24,012 cow alerts were generated (eating time, $\mathrm{n}=9,543$; lying time, $\mathrm{n}=$ 9,777 ; activity, $\mathrm{n}=1,590$; or a combination of behaviors, $\mathrm{n}=3,102$ ). Only $8 \%$ of the alerts were doubted by the producer. Although $55 \%$ of alerts were perceived to be true, producers visually assessed cows based on only $21 \%$ of the alerts with a large variation between farms (2 to $45 \%$ of the alerts visually assessed). Producers were more likely to completely ignore alerts over time. Producers were more likely to perceive cow alerts to be
\end{abstract}

Received May 1, 2019.

Accepted September 27, 2019.

*Corresponding author: eeckelka@utk.edu true and visually check cows when $\leq 20$ alerts occurred per day, cows were fresh or in early lactation, alerts occurred during the work week, or when cow alerts were for eating time, activity, or a combination of multiple behaviors. Behavioral disease alerts must be improved and correspond to an actionable change for producers to use them. Incorporating herd management software, creating and managing alerts by lactation stage, and focusing on behaviors producers already find useful could improve future alert utilization.

Key words: precision dairy technology, disease alert, transition cow disorder

\section{INTRODUCTION}

Precision dairy management (PDM) technologies are real-time monitors that supplement the eyes and ears of the farmer and improve individual cow management (Wathes et al., 2008; Berckmans, 2015). Precision dairy management technologies can be wearable, incorporated into the milking system, stand-alone, or part of the herd management software (Borchers and Bewley, 2015; Bewley et al., 2017). Wearable PDM (leg, neck, or ear attached) have been validated to accurately characterize activity (steps/d), lying time, standing time, walking time, eating time, and rumination time (Borchers et al., 2016; Van Erp-Van der Kooj et al., 2016). Changes in these behaviors have been linked to estrus (Farris, 1954; Fricke et al., 2014; Dolecheck et al., 2016), calving (Bar and Solomon, 2010; Borchers et al., 2017), and illness (Jawor et al., 2012; Itle et al., 2015; Liboreiro et al., 2015; Stangaferro et al., 2016a,b,c). Through behavioral changes, PDM could predict or detect illness events.

Precision dairy technologies used primarily for estrus detection have been explored for other purposes, including disease detection (Rutten et al., 2013; Eckelkamp, 2019). The primary behaviors used for disease detection have been rumination (Bar and Solomon, 2010; Stangaferro et al., 2016a,b,c), neck activity (Liberati and Zappavigna, 2009; Stangaferro et al., 2016a,b,c), step activity (Edwards and Tozer, 2004; Mazrier et al., 2006; Miekley et al., 2012), and lying time (Ito et 
Table 1. Herd characteristics of the 4 participating farms

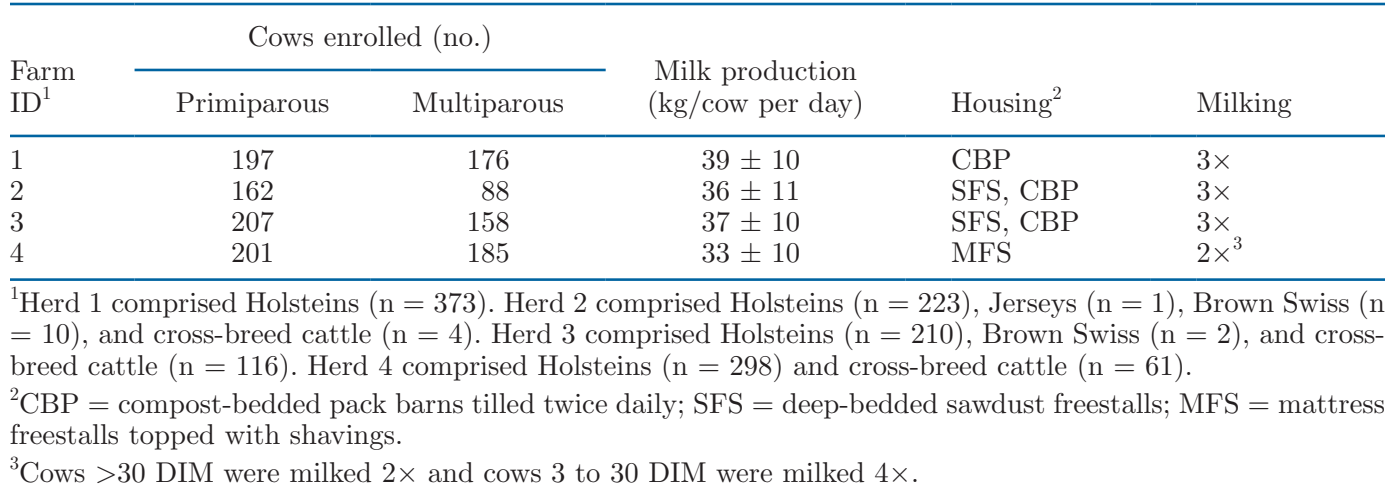

al., 2010). However, only Mazrier et al. (2006) used a threshold of detection to actively choose cows to evaluate for lameness. Similarly, to date, Hogeveen et al. (2013) have been the only researchers to categorize producer feedback on PDM-generated alerts.

Poor usability and information overload have been listed as leading reasons why PDM have not been adopted (Huirne et al., 1997; Russell and Bewley, 2013; Borchers and Bewley, 2015). Producers were overwhelmed by the vast information provided by systems and desired user-friendly technology and more training on technology implementation (Jago et al., 2013; Russell and Bewley, 2013; Borchers and Bewley, 2015). Hogeveen et al. (2013) witnessed the reality of information overload with an automated milking system. Of 421 mastitis alerts generated by the system, producers checked cows for only $3 \%$ of the alerts, although $40 \%$ corresponded to a mastitis event (Hogeveen et al., 2013). Producers did not follow up on alerts if no flakes or clots were on the filter sock ( $28 \%$ of alerts), milk production did not decrease ( $12 \%$ of alerts), cows were repeatedly on the list ( $10 \%$ of alerts), or they had no time $(10 \%$ of alerts). The low percentage of data perceived as useful by producers emphasized the need for more research on producers' use of PDM-generated alerts. More on-farm evaluations are needed to provide producers and manufacturers with more informationbased decisions in technology adoption and creation (Wathes et al., 2008; Jago et al., 2013).

Long-term evaluation of PDM-generated disease alerts is needed to identify how producers incorporate these systems into their daily management and perceive alert usefulness. The objective of this study was to quantify the usefulness of PDM technology-generated alerts for dairy producers. Usefulness was defined as alerts that producers perceived to be true and, more specifically, that led producers to visually assess cows. The hypotheses were that (1) producer perception of usefulness would decrease over time if unactionable alerts were created, (2) usefulness would be greater for behaviors that producers already associated with disease, and (3) usefulness would be greater in cows that producers were already monitoring, such as cows at higher risk for disease.

\section{MATERIALS AND METHODS}

Data were collected from 4 cooperating Kentucky dairy farms from October 5, 2015, to October 30, 2016. Producers interested in purchasing a new precision dairy technology system were approached in October 2014. Four producers agreed to purchase the technology, participate in the study, and evaluate daily technologygenerated herd health reports. Each farm was visited twice weekly for a total of 104 visits. All farms, herds, and producers were assigned an identifying number (1-4), and all herds in the study were enrolled in DHI. See Table 1 for complete herd descriptions.

Six months before study initiation, the entire lactating herd for each farm was equipped with a triaxial accelerometer $(70 \times 40 \times 72 \mathrm{~mm}, 108 \mathrm{~g}$; attached to the right or left rear leg with a thermoplastic polyurethane Nedap leg strap) measuring activity (steps/d) and lying time $(\mathrm{min} / \mathrm{d})$ as well as a triaxial accelerometer $(142 \times$ $80 \times 45 \mathrm{~mm}, 290 \mathrm{~g}$; attached around the neck) with a fully adjustable collar measuring eating time ( $\mathrm{min} / \mathrm{d}$; CowWatch). Both the leg and neck tri-accelerometer technologies were manufactured by Nedap Livestock Management (Groenlo, the Netherlands) and marketed by Alta Genetics Inc. (Watertown, WI). The technologies were validated for eating (neck tag only), lying (leg tag only), standing (leg tag only), walking (leg tag only), and activity (steps/d; leg tag only) characterization by Van Erp-Van der Kooj et al. (2016). Any new cows without technologies entering the lactating herd had tags attached at or around calving. The tags 
sent their respective information to a wireless reader (located in the holding pen with a 1,000-m wireless radius) every $15 \mathrm{~min}$ as the number of seconds a behavior (lying or eating time) occurred or the number of steps taken within that 15 -min interval. If the reader was out of range of the tags, data were stored for $24 \mathrm{~h}$ within the tag, and at each 15-min interval the tag attempted to connect with the reader again. Once a connection was established, all stored data were transferred to the reader.

\section{Data Collection}

Performance records from DHI, including bred and heat dates, DIM, parity, and number of lactating animals in the herd, were collected with the permission of participating producers. A Hobo U23 Series Pro v2 Logger (Onset, Cape Cod, MA) was used to collect barn temperature and humidity data. The Hobo logger was placed near the center of the primary housing barn above the height easily reached by cows and out of the direct airflow of fans. Data were offloaded weekly.

Herd Behavior Alert Report. A technologygenerated health report was created daily for every farm. Alert creation was based on a decrease of $\geq 30 \%$ from a cow's previous 10-d mean total daily activity (steps/d), lying time, or eating time. Until a full $10 \mathrm{~d}$ of data were collected on a cow, no alerts were created. The web-based interface presented alerts in the "health and management" alert list as cow, DIM, group, eating alert, lying alert, and activity alert. Only cows with $\geq 1$ alert were shown on the list. On May 11, 2016, a software update occurred for all farms. No changes in how alerts were calculated occurred, but the graphics in the interface changed and cows in estrus were no longer shown on the alert list. The 2 versions were included in evaluations to determine perceived changes in usefulness over time (version 1: October 5, 2015, to May 11, 2016; version 2: May 12, 2016, to October 31, 2016). Producers were asked to provide feedback on each cow alert that occurred on the list each day. A cow alert could contain a single change in activity, lying time, or eating time and any combination of the single changes.

Alert Categorization and Herd Health. To assess the perceived usefulness of cow alerts, producers recorded the perceived accuracy of an alert and the follow-up actions taken. Producers had the option to categorize alerts in an online Google form (Google LLC, Mountain View, CA). Only 1 producer used the online Google form. Three producers printed off the daily alert list and manually recorded the category and the reason for categorization, corresponding to the same setup as the Google form. Producers were asked to select only 1 category and 1 reason for choosing the category per cow alert (Table 2 and Figure 1; adapted from Hogeveen et al., 2013). A sample decision tree is provided in Figure 1 . When a cow alert occurred, a producer either would provide information on how the alert was used (categorized as Evaluated) or would not (categorized as NotEvaluated). Evaluated alerts could fall into 3 overall categories: (1) the cow alert was perceived to be true and the cow was visually checked (CowCheck), (2) the cow alert was perceived to be true, but the cow was not visually checked because of the alert (NoAction), or (3) the cow alert was doubted and the cow was not visually checked (AlertDoubted).

Alerts categorized as CowCheck were further divided into 5 reasons why the producer chose the category: (1) cow was visually sick and treated, (2) cow was visually sick and not treated, (3) cow was not visually sick and treated, (4) cow was not visually sick and not treated, and (5) other. Occasionally, producers provided written responses for "other" that included pen changes, calving, and estrus.

Alerts categorized as NoAction were further divided into 9 reasons why the producer chose the category: (1) the behavioral change from a cow's normal behavior did not concern the producer, (2) the cow underwent a pen change or dry-off, (3) the cow underwent a veterinary or pregnancy check or hoof trimming, (4) too many cows were currently being treated, (5) the cow had already been designated as a cull cow, (6) the cow was repeatedly on the alert list, (7) the cow was in estrus, (8) the producer had no time to visually check the cows, and (9) other. Occasionally, producers provided written responses for "other" that included estrus, weather changes, and feed changes.

Alerts categorized as AlertDoubted were further divided into 9 reasons why the producer chose the category: (1) the alert was not considered to represent a true behavioral change; (2) the cow underwent a pen change or dry-off; (3) the cow underwent a veterinary or pregnancy check or hoof trimming; (4) the cow had been previously checked and was not ill; (5) the ear or neck tag was defective, removed, or lost; (6) the cow was repeatedly on the alert list; (7) the cow was in estrus; (8) the producer had no time to visually check the cows; or (9) other. Occasionally, producers provided written responses for "other" that included estrus, weather changes, and feed changes.

If multiple reasons were selected (i.e., veterinary check and other), the reason providing the most information was selected (i.e., veterinary check). The similarities between reasons for NoAction and AlertDoubted were included to keep producers from choosing one reason and categorizing alerts based on the reason. Choosing 
Eckelkamp and Bewley: ON-FARM USE OF DISEASE ALERTS

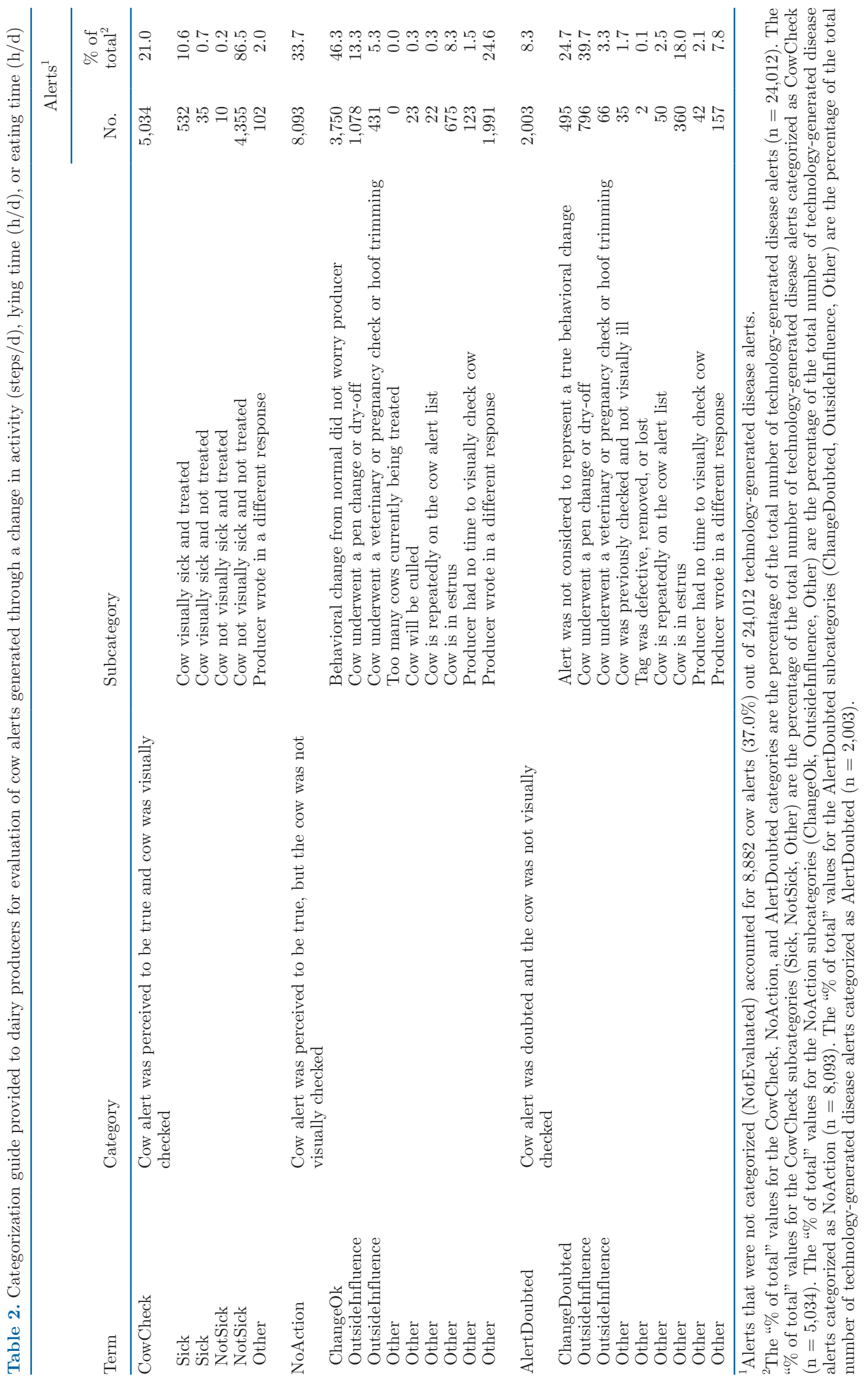




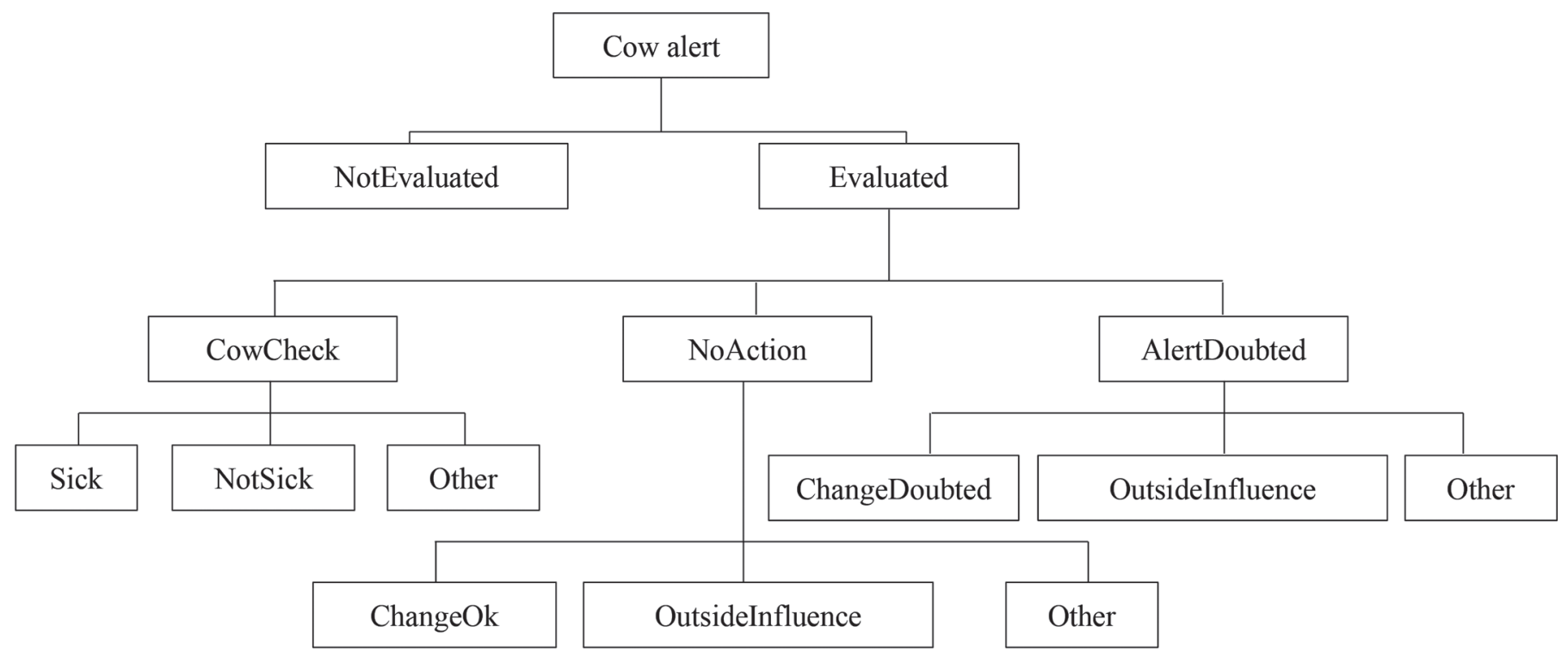

Figure 1. Decision tree for producer evaluation of technology-generated behavior alerts. Cow alerts were generated when a cow's lying time, eating time, activity (steps/d), or any combination decreased 30\% or more from her 10-d moving average. Cow alerts were collected using wearable precision dairy technologies from October 5, 2015, to October 30, 2016. See Table 2 for a full explanation of cow alert categorization. NotEvaluated indicated that cow alerts occurred but no producer feedback was given. Evaluated indicated that cow alerts occurred and producer feedback was given. Evaluated cow alerts were categorized as CowCheck (alert was perceived to be true and the cow was visually checked), NoAction (alert was perceived to be true, but the cow was not visually checked because of the alert), and AlertDoubted (alert was doubted and the cow was not visually checked).

an overall category (CowCheck, NoAction, or AlertDoubted) captured how the producer perceived that cow alert's usefulness and then allowed them to select why the cow alert was categorized as CowCheck, NoAction, or AlertDoubted. The same individual on each farm categorized alerts throughout the study.

\section{Statistical Analyses}

Data Cleaning. Because dairy producers evaluated only lactating dairy cows, the data set was limited to lactating cows. Of the 1,197 cows in all herds with recorded disease events or disease alerts, 26 cows were removed from the data set because tags were assigned to incorrect cows, leaving 1,171 cows.

The FREQ procedure of SAS 9.4 (SAS Institute Inc., Cary, NC) with a chi-squared analysis was used to assess category and reason distribution across and within herds. Reasons that accounted for $\leq 10 \%$ of cow alert categorizations within CowCheck, NoAction, or AlertDoubted were combined with similar variables to improve odds ratio calculation (Table 2). Within CowCheck, reasons were condensed to (1) Sick (visually sick and treated or visually sick and not treated), (2) NotSick (not visually sick and treated or not visually sick and not treated), and (3) Other (producer wrote in another response).
Within NoAction, reasons were condensed to (1) ChangeOk (the behavioral change from normal behavior did not concern the producer), (2) OutsideInfluence (cow underwent a pen change, dry-off, veterinary or pregnancy check, or hoof trim), and (3) Other (too many cows currently being treated, cow would be culled, cow repeatedly on the list, cow was in estrus, producer had no time to visually check cows, or producer wrote in a different response).

Within AlertDoubted, reasons were condensed to (1) ChangeDoubted (the alert was not considered to represent a true behavior change), (2) OutsideInfluence (cow underwent a pen change, dry-off, veterinary or pregnancy check, or hoof trim), and (3) Other (cow previously checked and not ill; tag was defective, removed, or lost; cow was repeatedly on the list; cow was in estrus; producer had no time to visually check cows; or producer wrote in a different response). When analyses within CowCheck, NoAction, or AlertDoubted were conducted, the data set was reduced to include only alerts categorized as CowCheck, NoAction, or AlertDoubted, respectively. Associated cow numbers are given as $(\mathrm{n}=X)$ throughout the text.

Univariate Analyses. A univariate analysis was conducted to test individual effects on alert categorization. Significant univariate effects $(P<0.05)$ were included in a multivariate model as fixed effects (Table 
3). Fixed effects tested individually were software version (version 1: October 5, 2015, to May 11, 2016; version 2: May 12, 2016, to October 31, 2016), day group, lactation stage, parity, alerts per day, behavior type, and heat stress. Day group was either weekday (Monday to Friday) or weekend (Saturday to Sunday). Lactation stage was fresh (0-29 DIM), early (30-100 DIM), or post-peak (>100 DIM). Parity group was first lactation, second lactation, or third or more lactation. Alerts per day were either $\leq 20$ alerts or $>20$ alerts. Alerts per day was divided based on the default number the health and management alert list displayed ( 20 cow alerts). Multiple pages had to be viewed or the default number of alerts shown had to be changed from 20 to 30,50 , or 100 to view $\geq 20$ cow alerts in a day. Behavior type triggering alerts was eating time, lying time, activity, or any combination of eating, lying, and activity. Heat stress was either present $(\geq 68$ maximum daily temperature-humidity index, THI) or absent $(<68$ maximum daily THI). Temperature-humidity index was calculated using Equation 1 (NOAA, 1976):

$$
\begin{aligned}
\mathrm{THI} & =\text { temperature, }{ }^{\circ} \mathrm{F}-\{0.55-[0.55 \\
& \times(\text { relative humidity } / 100)]\} \\
& \times\left(\text { temperature },{ }^{\circ} \mathrm{F}-58.8\right) .
\end{aligned}
$$

A generalized linear mixed model (GLIMMIX procedure of SAS 9.4) with binary distribution and logit link was used to test fixed effects on the probability of a cow alert being categorized as Evaluated or NotEvaluated. The model contained 24,012 observations on 1,171 cows across 4 farms. The random effect of cow nested within farm was included in all models. Repeated measures of cow nested within farm over the study were also included in all models.

A generalized linear mixed model with multinomial distribution and generalized logit link was used to test fixed effects on the cow alert categorization probability. Across CowCheck, NoAction, and AlertDoubted, the model contained 15,130 observations on 1,121 cows across 4 farms. Across Sick, NotSick, and Other within CowCheck, the model contained 5,034 observations on 753 cows across 4 farms. Across ChangeOk, OutsideInfluence, and Other within NoAction, the model contained 8,093 observations on 1,050 cows across 4 farms. Across ChangeDoubted, OutsideInfluence, or Other within AlertDoubted, the model contained 2,003 observations on 560 cows across 4 farms. The random effect of cow nested within farm was included in the model. Repeated measures of cow nested within farm over the study were also included in the model.

Multivariate Analysis-Any Feedback. A generalized linear mixed multivariate analysis was conducted to determine the effects of significant univariate variables in combination on alert categorization (GLIMMIX procedure of SAS 9.4). A generalized linear mixed model with binary distribution and logit link was used to test fixed effects on alert categorization as Evaluated (producer provided use information) or NotEvaluated (producer provided no use information). Fixed effects were software version, day group, lactation stage, heat stress, alerts per day, and behavior type. The model contained 24,012 observations on 1,171 cows across 4 farms. The random effect of cow nested within farm was included in the model. Repeated measures of cow nested within farm over the study were also included in the model. Odds ratios were calculated comparing Evaluated alerts against NotEvaluated alerts for each fixed effect.

Multivariate Analysis-Evaluated Alert Categorization. A generalized linear mixed model with multinomial distribution and generalized logit link was used to test significant univariate variable fixed effects on alert categorization as CowCheck, NoAction, or AlertDoubted. Significant fixed effects were software version, lactation stage, heat stress, alerts per day, and behavior type. The model contained 15,130 observations on 1,121 cows across 4 farms. The random effect of cow nested within farm was included in the model. Repeated measures of cow nested within farm over the study were also included in the model. Odds ratios were calculated comparing CowCheck against NoAction, CowCheck against AlertDoubted, and NoAction against AlertDoubted for each fixed effect.

Multivariate Analysis-Reasons for Categorization: CowCheck. A generalized linear mixed model with multinomial distribution and generalized logit link was used to test significant univariate variable fixed effects to compare Sick, NotSick, and Other as reasons for categorizing cow alerts as CowCheck. Significant fixed effects were day group, lactation stage, alerts per day, and behavior type. The model contained 5,034 observations on 753 cows across 4 farms. The random effect of cow nested within farm was included in the model. Repeated measures of cow nested within farm over the study were also included in the model. Odds ratios were calculated comparing NotSick against Sick, Sick against Other, and NotSick against Other for each fixed effect.

Multivariate Analysis-Reasons for Categorization: NoAction. A generalized linear mixed model with multinomial distribution and generalized logit link was used to test significant univariate variable fixed effects to compare ChangeOk, OutsideInfluence, and Other as reasons for categorizing cow alerts as NoCheck. Significant fixed effects were software version, day group, lactation stage, heat stress, alerts per day, 
Table 3. Univariate categorical influencers of producer categorization of cow alerts generated by wearable precision dairy technologies

\begin{tabular}{|c|c|c|}
\hline Producer categorization & Influencer $^{1}$ & $P$-value \\
\hline \multicolumn{3}{|l|}{ Evaluated or NotEvaluated $^{2}$} \\
\hline & Software version & $<0.01$ \\
\hline & Day group & $<0.01$ \\
\hline & Lactation stage & $<0.01$ \\
\hline & Parity group & 0.84 \\
\hline & Heat stress & $<0.01$ \\
\hline & Alerts per day & $<0.01$ \\
\hline & Behavior alerted & $<0.01$ \\
\hline \multicolumn{3}{|l|}{ CowCheck, ${ }^{3}$ NoAction, ${ }^{4}$ or AlertDoubted ${ }^{5}$} \\
\hline & Software version & $<0.01$ \\
\hline & Day group & $<0.06$ \\
\hline & Lactation stage & $<0.01$ \\
\hline & Parity group & $<0.01$ \\
\hline & Heat stress & $<0.01$ \\
\hline & Alerts per day & $<0.01$ \\
\hline & Behavior alerted & $<0.01$ \\
\hline \multicolumn{3}{|l|}{ Within CowCheck ${ }^{6}$} \\
\hline & Software version & 0.11 \\
\hline & Day group & $<0.01$ \\
\hline & Lactation stage & 0.04 \\
\hline & Parity group & 0.04 \\
\hline & Heat stress & 0.16 \\
\hline & Alerts per day & $<0.01$ \\
\hline & Behavior alerted & $<0.01$ \\
\hline \multicolumn{3}{|l|}{ Within NoAction ${ }^{7}$} \\
\hline & Software version & $<0.01$ \\
\hline & Day group & $<0.01$ \\
\hline & Lactation stage & $<0.01$ \\
\hline & Parity group & $<0.01$ \\
\hline & Heat stress & $<0.01$ \\
\hline & Alerts per day & $<0.01$ \\
\hline & Behavior alerted & $<0.01$ \\
\hline \multicolumn{3}{|l|}{ Within AlertDoubted $^{8}$} \\
\hline & Software version & $<0.01$ \\
\hline & Day group & $<0.01$ \\
\hline & Lactation stage & $<0.01$ \\
\hline & Parity group & $<0.01$ \\
\hline & Heat stress & $<0.01$ \\
\hline & Alerts per day & $<0.01$ \\
\hline & Behavior alerted & $<0.01$ \\
\hline
\end{tabular}

${ }^{1}$ Only influencers with $P<0.05$ were included in a multivariate GLIMMIX procedure. Software version was grouped as version 1 (pre-May 2016) or version 2 (post-May 2016). The software was updated on May 11, 2016, to a visually different interface, and cows in estrus were not included on the health and management list. Day group was a weekday (Monday to Friday) or weekend (Saturday to Sunday). Lactation stage was grouped as fresh ( $\leq 30$ DIM), early lactation (31-99 DIM), or post-peak lactation ( $\geq 100$ DIM). Parity group was first, second, or $\geq$ third lactation. Heat stress was grouped as temperature-humidity index $\geq 68$ or $<68$. Alerts per day were grouped as $\leq 20$ or $>20$ cow alerts on the list per day. Twenty cow alerts corresponded to the default number displayed on the health and management list. Behavior alerted referred to eating time, lying time, or activity (steps/d) and any 2- or 3-way combination of the behaviors that decreased below the predetermined threshold and triggered an alert to be created on the health and management list.

${ }^{2}$ Evaluated indicates that cow alerts occurred and producer feedback was given. Evaluated cow alerts were categorized as CowCheck, NoAction, and ChangeDoubted. NotEvaluated indicates that cow alerts occurred but no producer feedback was given.

${ }^{3}$ The cow alert was perceived to be true and the cow was visually checked.

${ }^{4}$ The cow alert was perceived to be true, but the cow was not visually checked because of the alert.

${ }^{5}$ The cow alert was doubted and the cow was not visually checked.

${ }^{6}$ Within the CowCheck category, Sick indicated that a cow was visually sick, NotSick indicated that a cow was not visually sick, and Other indicated that the producer wrote in their own response. Common responses included calving, pen change, and estrus (Table 2; Figure 1).

${ }^{7}$ Within the NoAction category, ChangeOk indicated that the behavioral change from normal did not worry the producer; OutsideInfluence indicated that the behavioral change was attributed to a pen change, dry-off, veterinary or pregnancy check, or hoof trimming; and Other indicated that the producer wrote in their own response. Common responses included estrus, weather changes, and the producer had no time to visually assess cows (Table 2; Figure 1). ${ }^{8}$ Within the AlertDoubted category, ChangeDoubted indicated that the alert was not considered to represent a true behavior change; OutsideInfluence indicated that the behavioral change was attributed to management change, including pen change, dry-off, veterinary or pregnancy check, or hoof trimming; and Other indicated that the producer wrote in their own response. Common responses included cow repeatedly on the list, weather changes, and the producer had no time to visually assess cows (Table 2; Figure 1). 
and behavior type. The model contained 8,093 observations on 1,050 cows across 4 farms. The random effect of cow nested within farm was included in the model. Repeated measures of cow nested within farm over the study were also included in the model. Odds ratios were calculated comparing ChangeOk against OutsideInfluence, ChangeOk against Other, and OutsideInfluence against Other for each fixed effect.

Multivariate Analysis-Reasons for Categorization: AlertDoubted. A generalized linear mixed model with multinomial distribution and generalized logit link was used to test significant univariate variable fixed effects to compare ChangeDoubted, OutsideInfluence, and Other as reasons for categorizing cow alerts as AlertDoubted. Significant fixed effects were software version, day group, lactation stage, heat stress, alerts per day, and behavior type. The model contained 2,003 observations on 560 cows across 4 farms. The random effect of cow nested within farm was included in the model. Repeated measures of cow nested within farm over the study were also included in the model. Odds ratios were calculated comparing OutsideInfluence against ChangeDoubted, OutsideInfluence against Other, and ChangeDoubted against Other for each fixed effect.

\section{RESULTS}

Overall, 24,012 cow alerts were generated from 1,171 cows. Herds 1, 2, 3, and 4 included $217 \pm 23,137 \pm 17$, $202 \pm 14$, and $230 \pm 14$ lactating cows over the study period, respectively. Of the 24,012 cow alerts, producers provided feedback on 15,130 cow alerts (Evaluated: $63 \%)$. The remaining cow alerts $(8,882)$ had no feedback associated with them (NotEvaluated). Cow alerts were caused by decreased eating time $(\mathrm{n}=9,543)$, lying time $(\mathrm{n}=9,777)$ and activity $(\mathrm{n}=1,590)$ or a combination of behaviors $(\mathrm{n}=3,102)$. Across herds, cow alert evaluation and categorization differed by producer (Table 4 ). The univariate analyses (Table 3 ) identified variables of interest for multivariate analyses of Evaluated versus NotEvaluated cow alerts, categorization of Evaluated cow alerts, and subcategorization of cow alerts.

\section{Overall Evaluation: Evaluated Versus NotEvaluated}

Software version, day group, lactation stage, heat stress, alerts per day, and behavior type all influenced alert evaluation $(P<0.01$, respectively; Table 3$)$ in the univariate analyses. Within the multivariate analysis, producers were more likely to evaluate alerts during version 1 , weekdays, and fresh and early lactation and when $\leq 20$ cow alerts occurred (Table 5). Producers were more likely to evaluate eating alerts over lying

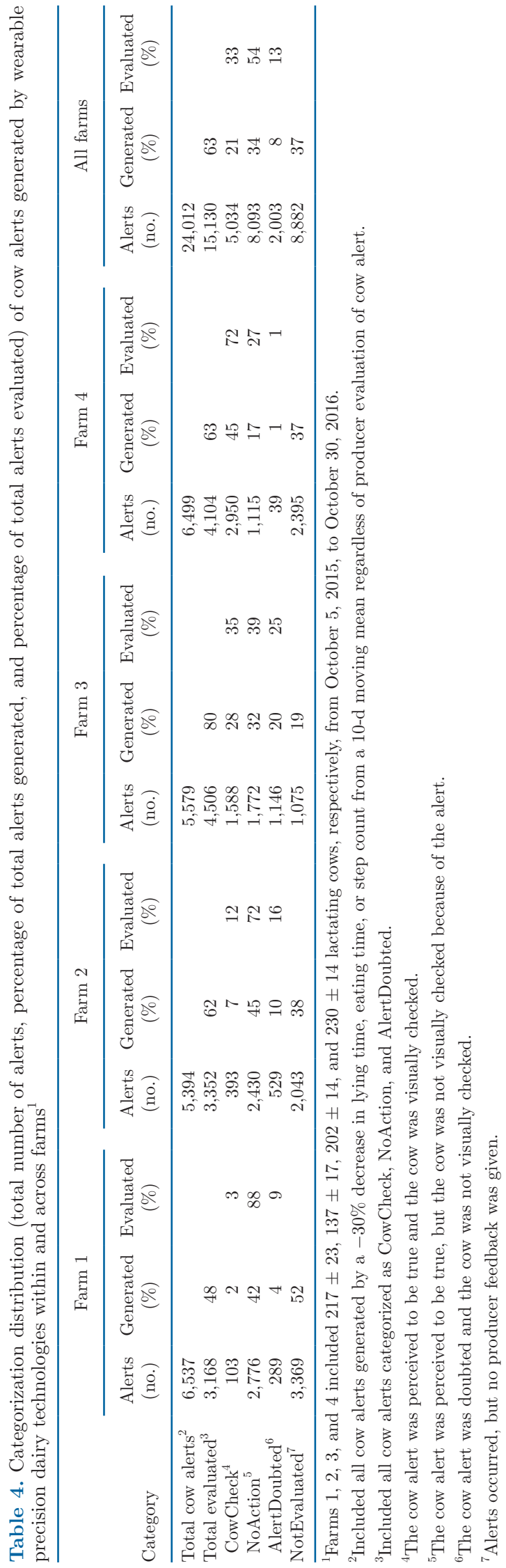


Table 5. Odds ratios and $95 \%$ confidence intervals for system, ${ }^{1}$ day group, ${ }^{2}$ lactation stage, ${ }^{3}$ alerts per day, ${ }^{4}$ and behavior alerted ${ }^{5}$ effects of dairy producer cow alert ${ }^{6}$ evaluation

\begin{tabular}{|c|c|c|c|c|c|}
\hline Categorization & Risk factor & Reference group & Odds ratio & $95 \% \mathrm{CI}^{8}$ & $P$-value \\
\hline & Version & Version 2 & 1.40 & $1.24-1.57$ & $<0.01$ \\
\hline & Early lactation & Post-peak lactation & 1.30 & $1.15-1.46$ & $<0.01$ \\
\hline & $\leq 20$ alerts $/ \mathrm{d}$ & $>20$ alerts $/ \mathrm{d}$ & 1.92 & $1.78-2.07$ & $<0.01$ \\
\hline & $\overline{\text { Combination }}$ & Lying & 1.14 & $1.01-1.29$ & $<0.01$ \\
\hline & Activity & Eating & 1.33 & $1.13-1.56$ & $<0.01$ \\
\hline & Activity & Lying & 1.45 & $1.23-1.70$ & $<0.01$ \\
\hline
\end{tabular}

${ }^{1}$ Software version was grouped as version 1 (pre-May 2016) or version 2 (post-May 2016). The software was updated on May 11, 2016, to a visually different interface, and cows in estrus were not included on the health and management list.

${ }^{2}$ Day group was weekday (Monday to Friday) or weekend (Saturday to Sunday).

${ }^{3}$ Lactation stage was grouped as fresh $(\leq 30$ DIM), early (31-99 DIM), or post-peak ( $\geq 100$ DIM) lactation.

${ }^{4}$ Alerts per day were grouped as $\leq 20$ or $>20$ cow alerts on the list per day; 20 cow alerts corresponded to the default number displayed on the health and management list.

${ }^{5}$ Behavior alerted referred to eating time, lying time, or activity (steps/d) and any 2- or 3-way combination of the behaviors that decreased below the predetermined threshold and triggered an alert to be created on the health and management list.

${ }^{6}$ Cow alerts were generated for a change in activity (steps/d), lying time (h/d), or eating time (h/d) collected by wearable precision dairy technologies.

${ }^{7}$ Heat stress was grouped as temperature-humidity index $\geq 68$ or $<68$. Heat stress was not a significant influencer of evaluating or not evaluating cow alerts $(P=0.71)$.

${ }^{8}$ Confidence intervals overlapping the null value (odds ratio $=1$ ) were not included in the table.

${ }^{9}$ Evaluated indicated that cow alerts occurred and producer feedback was given. Evaluated cow alerts were categorized as CowCheck (cow alert was perceived to be true and cow was visually checked), NoAction (cow alert was perceived to be true, but the cow was not visually checked because of the alert), and AlertDoubted (cow alert was doubted and cow was not visually checked). NotEvaluated indicated that cow alerts occurred, but no producer feedback was given.

alerts, combinations over lying alerts, and activity over combination, eating, and lying alerts (Table 5).

\section{Alert Usefulness: CowCheck Versus NoAction Versus AlertDoubted}

To assess perceived usefulness, producers categorized each cow alert as CowCheck, NoAction, and AlertDoubted. Software version, lactation stage, parity group, heat stress, alerts per day, and behavior alerted all influenced perceived usefulness $(P<0.01$; Table 3$)$. Producers were more likely to categorize cow alerts as CowCheck in version 2 when $\leq 20$ cow alerts occurred (Table 6). Producers were more likely to physically follow up on eating alerts or combinations of multiple alerts than on activity or lying alerts (Table 6). Conversely, producers were more likely to categorize alerts as NoAction instead of AlertDoubted when $>20$ cow alerts occurred.

\section{Subcategorization: CowCheck}

To assess perceived accuracy of CowCheck alerts, producers provided further categorization of the cows as Sick, NotSick, and Other. Day group, lactation stage, parity group, alerts per day, and behavior alerted influenced perceived accuracy $(P<0.05$; Table 3$)$. Producers were more likely to perceive accuracy when cow alerts occurred during weekdays and when $>20$ cow alerts occurred (Table 7). Producers were more likely to perceive accuracy for eating alerts or combinations of multiple alerts than for activity or lying alerts (Table 7).

\section{Subcategorization: NoAction}

To determine why alerts were not acted on (NoAction), producers further categorized cow alerts as ChangeOk, OutsideInfluence, and Other. Software version, day group, lactation stage, parity group, heat stress, alerts per day, and behavior alerted all influenced why cow alerts were not acted on $(P<$ 0.01; Table 3). Producers were more likely to attribute cow alerts to OutsideInfluence versus ChangeOk in version 1, during fresh or early lactation, when cows were not experiencing heat stress, when $>20$ cow alerts occurred, and when combinations of multiple alerts, eating, or activity alerts occurred (Table 8). Producers were more likely to attribute cow alerts to ChangeOk versus Other on weekends, during fresh 
Table 6. Odds ratios and $95 \%$ confidence intervals for system, ${ }^{1}$ alerts per day, ${ }^{2}$ and behavior alerted ${ }^{3}$ effects on dairy producer cow alert ${ }^{4}$ evaluation ${ }^{5}$

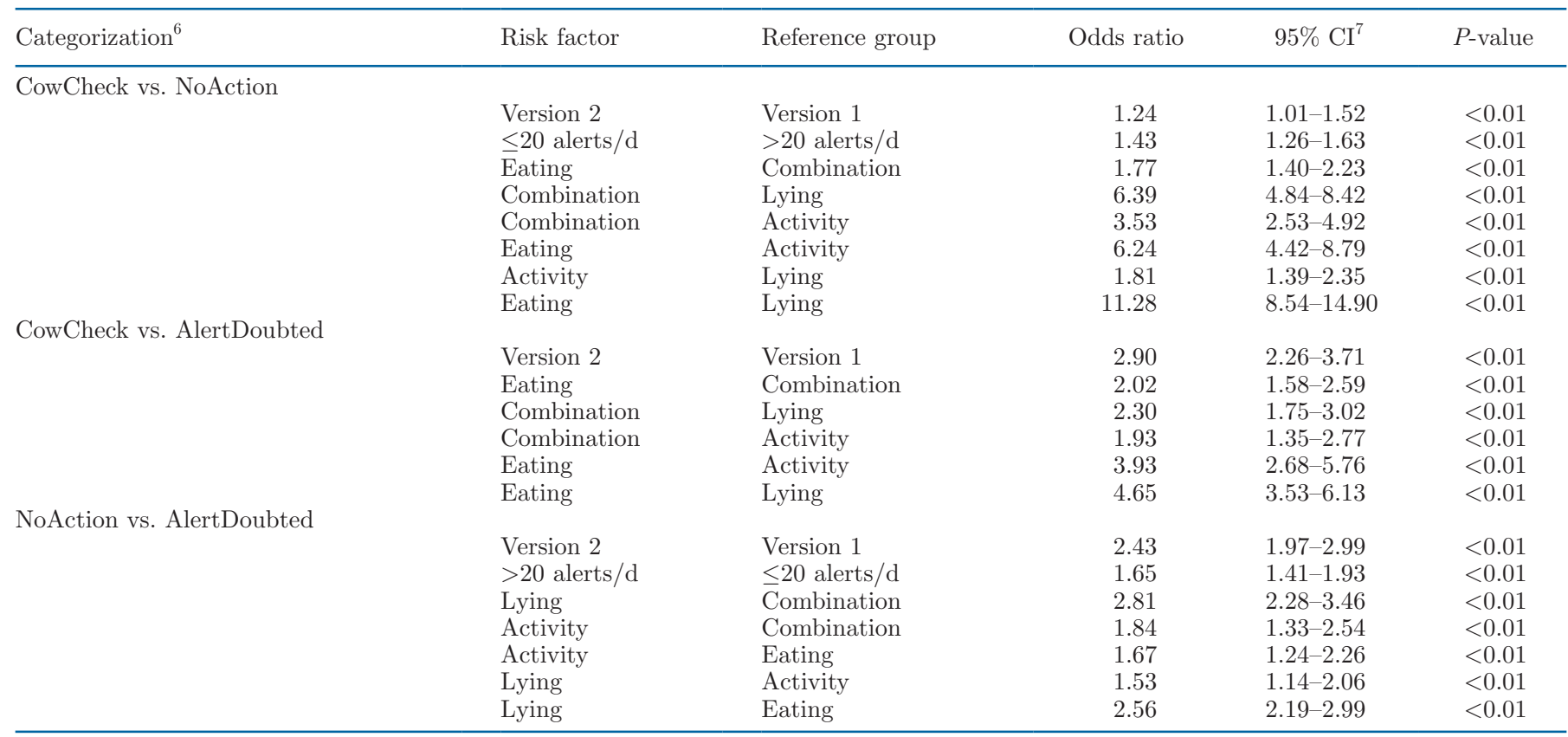

${ }^{1}$ Software version was grouped as version 1 (pre-May 2016) or version 2 (post-May 2016). The software was updated on May 11, 2016, to a visually different interface, and cows in estrus were not included on the health and management list.

${ }^{2}$ Alerts per day were grouped as $\leq 20$ or $>20$ cow alerts on the list per day. Twenty cow alerts corresponded to the default number displayed on the health and management list.

${ }^{3}$ Behavior alerted referred to eating time, lying time, or activity (steps/d) and any 2- or 3-way combination of the behaviors that decreased below the predetermined threshold and triggered an alert to be created on the health and management list.

${ }^{4}$ Cow alerts were generated for a change in activity (steps/d), lying time $(\mathrm{h} / \mathrm{d})$, or eating time $(\mathrm{h} / \mathrm{d})$ collected by wearable precision dairy technologies.

${ }^{5}$ Lactation stage was grouped as fresh ( $\leq 30$ DIM), early (31-99 DIM), or post-peak ( $\geq 100$ DIM) lactation. Stage of lactation was not a significant influencer of CowCheck, NoAction, or AlertDoubted $(P=0.08)$. Parity group was first, second, and third or more lactation. Parity was not a significant influencer of CowCheck, NoAction, or AlertDoubted $(P=0.26)$. Heat stress was grouped as temperature-humidity index $\geq 68$ or $<68$. Heat stress was not a significant influencer of CowCheck, NoAction, or AlertDoubted $(P=0.97)$.

${ }^{6}$ CowCheck indicated that the cow alert was perceived to be true and the cow was visually checked. NoAction indicated that the cow alert was perceived to be true, but the cow was not visually checked because of the alert. AlertDoubted indicated that the cow alert was doubted and the cow was not visually checked.

${ }^{7}$ Confidence intervals overlapping the null value (odds ratio $=1$ ) were not included in the table.

lactation, when cows were heat stressed, when $\leq 20$ cow alerts occurred, and when lying or activity alerts occurred (Table 8).

\section{Subcategorization: AlertDoubted}

To determine why alerts were not believed to be true (AlertDoubted), producers further categorized cow alerts into ChangeDoubted, OutsideInfluence, and Other. Software version, day group, lactation stage, parity group, heat stress, alerts per day, and behavior alerted all influenced why cow alerts were not acted on $(P<0.01$; Table 3$)$. Producers were more likely to categorize cow alerts as OutsideInfluence versus ChangeDoubted in version 2, during fresh lactation, when cows were not heat stressed, and when combinations of multiple alerts, lying, or activity alerts occurred (Table 9). Producers were more likely to categorize cow alerts as ChangeDoubted versus Other in version 2, during weekends, during fresh lactation, when $\leq 20$ cow alerts occurred, and when combinations of multiple alerts or eating alerts occurred (Table 9).

\section{DISCUSSION}

Within our study, producers provided detailed feedback on perceived daily usefulness and accuracy of PDM-generated cow alerts. Sensitivity, specificity, accuracy, and balanced accuracy were explored by E. Eckelkamp (unpublished data). Overall, producers 
Table 7. Odds ratios and $95 \%$ confidence intervals for day group, ${ }^{1}$ alerts per day, ${ }^{2}$ and behavior alerted ${ }^{3}$ effects on dairy producer cow alert ${ }^{4}$ evaluation when the cow alert was perceived to be true and the cow was visually checked based on the alert ${ }^{5}$

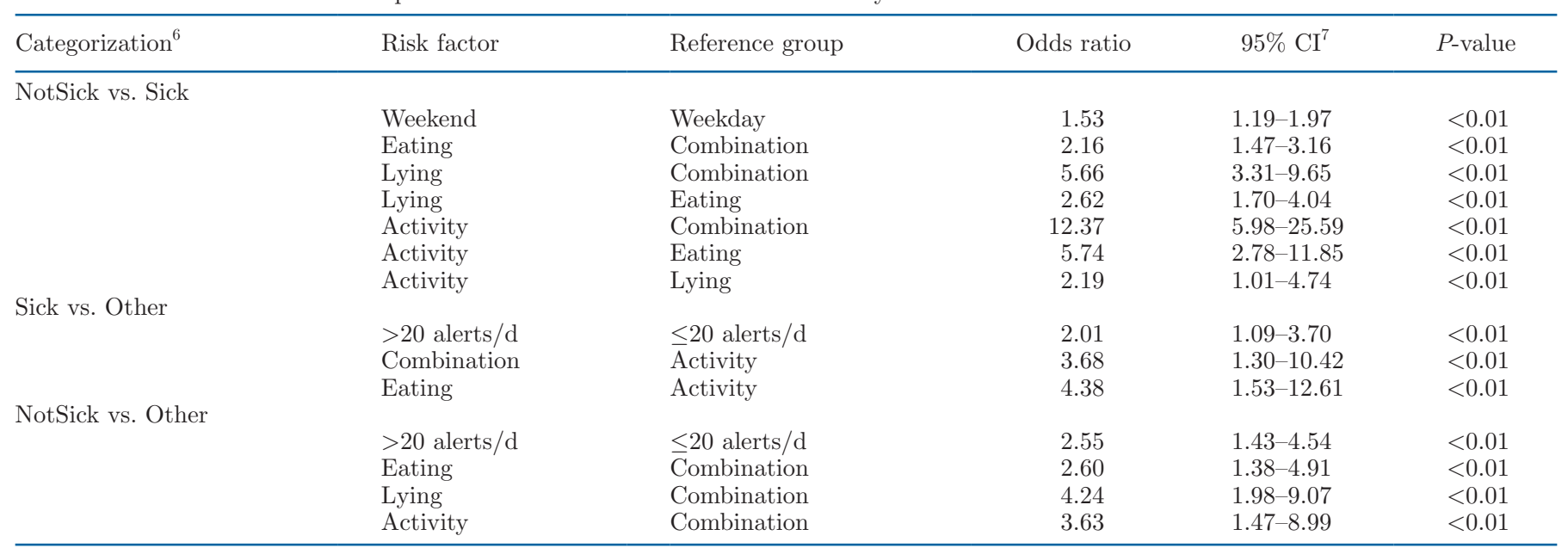

${ }^{1}$ Day group was weekday (Monday to Friday) or weekend (Saturday to Sunday).

${ }^{2}$ Alerts per day were grouped as $\leq 20$ or $>20$ cow alerts on the list per day. Twenty cow alerts corresponded to the default number displayed on the health and management list.

${ }^{3}$ Behavior alerted referred to eating time, lying time, or activity (steps/d) and any 2- or 3-way combination of the behaviors that decreased below the predetermined threshold and triggered an alert to be created on the health and management list.

${ }^{4}$ Cow alerts were generated for a change in activity (steps/d), lying time (h/d), or eating time (h/d) collected by wearable precision dairy technologies.

${ }^{5}$ Lactation stage was grouped as fresh ( $\leq 30$ DIM), early (31-99 DIM), or post-peak ( $\geq 100$ DIM) lactation. Lactation stage was not a significant influencer of NotSick, Sick, or Other $(P=0.07)$. Parity group was first, second, and third or more lactation. Parity group was not a significant influencer of NotSick, Sick, or Other $(P=0.09)$.

${ }^{6}$ Within the cow alert category CowCheck (alert perceived to be true and cow was visually checked), NotSick indicated that a cow was not visually sick. Sick indicated that a cow was visually sick. Other indicated that the producers wrote in their own response; common responses included calving, pen change, and estrus (Table 2; Figure 1).

${ }^{7}$ Confidence intervals overlapping the null value (odds ratio $=1$ ) were not included in the table.

provided evaluation on $63 \%$ of cow alerts but actively checked only 21\%. Similarly, Hogeveen et al. (2013) monitored a snapshot of mastitis alerts created by an automated milking system and found that only $3 \%$ of the 421 alerts were checked by producers. A disease alert system is only as beneficial as the producers find it, and the producers in Hogeveen et al. (2013) and our study found a small percentage of alerts usable. Within our study, a popular response was ChangeOk (behavior change from normal did not worry the producer). Similarly, the most popular reason for not visually checking a cow for mastitis was no flakes or clots were found on the filter (28\% of responses; Hogeveen et al., 2013). Admittedly, producers missed a large portion of mastitis events (74\%) in the Hogeveen et al. (2013) study, but in our study a low disease detection sensitivity (proportion of true positives) was associated with eating, lying, and activity (steps/d) alerts ( $31 \pm 4$ to $42 \pm 3$ sensitivity; E. Eckelkamp, unpublished data). The low sensitivity in our study coupled with the producer categorization of alerts as NoAction indicated that too many alerts without a clear follow-up action or health-related issue were occurring.
Within our study, alert usefulness varied by the producer. Some producers rarely visually checked cows based on alerts, whereas others preferred to check most of the cows. Differences in usefulness did not necessarily mean that the system performed differently on these farms. Hogeveen et al. (2013) reported similar differences, with one producer routinely checking an automatically generated mastitis alert list 10 times per day and another checking the list 2 to 3 times per week. Producer management style could also have influenced producer opinion. Farms 3 and 4 relied on nonfamily labor to conduct daily tasks, allowing owners to spend time evaluating cow alert lists and visually assessing cows. Farms 1 and 2 had limited nonfamily labor, relying instead on family labor to run the farm. Reliance on family labor limited the amount of daily time owners could spend evaluating alerts and checking cows because they were busy with other on-farm tasks (J. Kiser, R. Mattingly, F. Rowe, and S. Sidebottom; personal communication).

Producers on farm 1 had the lowest number of alerts categorized as CowCheck (2\%). An elevated feed wagon was used to feed fresh cows on farm 1 . The feed wagon 
design (slanted head entrances) pressed against the neck collar and prevented the triaxial accelerometer from registering head movement, resulting in eating attention for all cows in that pen. Over time, the alerts on cows within that pen were categorized as NoAction and written in as "fresh cows." Including producers in technology development could help pinpoint issues such as this before farm implementation and improve enduser confidence in the technology (Wathes et al., 2008).

\section{System}

The longer the system was in place, the less likely dairy producers were to give feedback on alerts (com-

Table 8. Odds ratios and $95 \%$ confidence intervals for system, ${ }^{1}$ day group, ${ }^{2}$ lactation stage,${ }^{3}$ heat stress, ${ }^{4}$ alerts per day, ${ }^{5}$ and behavior alerted ${ }^{6}$ effects on dairy producer cow alert ${ }^{7}$ evaluation when cow alerts were perceived to be true, but the cow was not visually checked because of the alert $^{8}$

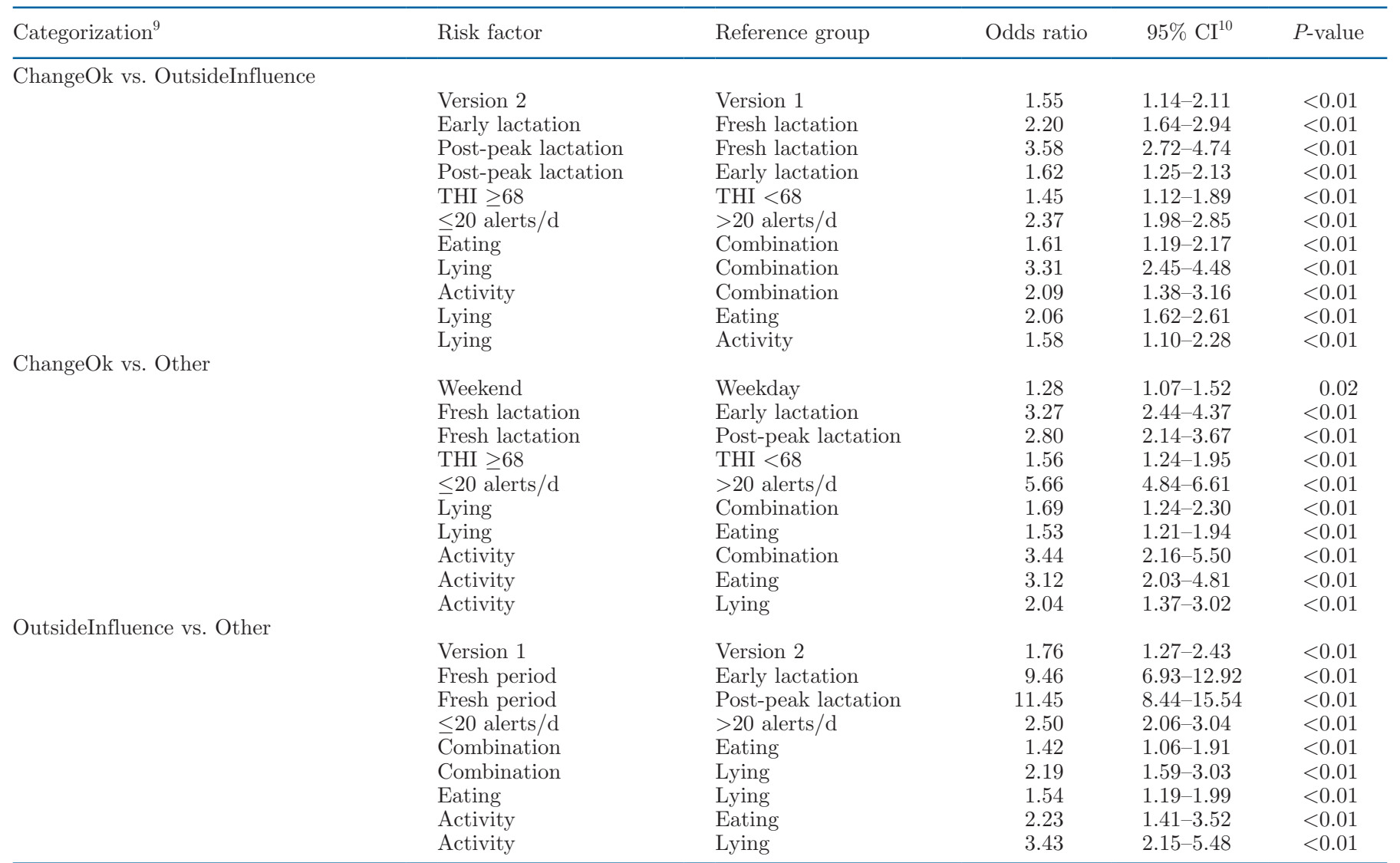

${ }^{1}$ Software version was grouped as version 1 (pre-May 2016) or version 2 (post-May 2016). The software was updated on May 11, 2016, to a visually different interface, and cows in estrus were not included on the health and management list.

${ }^{2}$ Day group was weekday (Monday to Friday) or weekend (Saturday to Sunday).

${ }^{3}$ Lactation stage was grouped as fresh ( $\leq 30$ DIM), early (31-99 DIM), or post-peak ( $\geq 100$ DIM) lactation.

${ }^{4}$ Heat stress was grouped as temperature-humidity index $\geq 68$ or $<68$.

${ }^{5}$ Alerts per day were grouped as $\leq 20$ or $>20$ cow alerts on the list per day. Twenty cow alerts corresponded to the default number displayed on the health and management list.

${ }^{6}$ Behavior alerted referred to eating time, lying time, or activity (steps/d) and any 2- or 3-way combination of the behaviors that decreased below the predetermined threshold and triggered an alert to be created on the health and management list.

${ }^{7}$ Cow alerts were generated for a change in activity (steps/d), lying time $(\mathrm{h} / \mathrm{d})$, or eating time $(\mathrm{h} / \mathrm{d})$ collected by wearable precision dairy technologies.

${ }^{8}$ Parity group was first, second, and third or more lactation. Parity group was not a significant influencer of ChangeOk, OutsideInfluence, or Other $(P=0.61)$.

${ }^{9}$ Within the cow alert category NoAction (cow alert was perceived to be true, but the cow was not visually checked because of the alert), ChangeOk indicated that the behavioral change from normal did not worry the producer. OutsideInfluence indicated that the behavioral change was attributed to a pen change, dry-off, veterinary or pregnancy check, or hoof trimming. Other indicated that the producers wrote in their own response; common responses included estrus, weather changes, and the producer had no time to visually assess cows (Table 2; Figure 1).

${ }^{10}$ Confidence intervals overlapping the null value (odds ratio $=1$ ) were not included in the table. 
paring Evaluated and NotEvaluated alerts). According to the producers participating in this study, lack of feedback could indicate that they were too busy, the system was not functioning correctly (lightning storms, lack of internet connection), or they were overloaded with alerts $(\geq 100$ alerts on the list per day; J. Kiser, R. Mattingly, F. Rowe, and S. Sidebottom; personal communication). Producers consistently provided cow alert feedback (CowCheck, NoAction, or AlertDoubted) at least weekly over the study. Producers 1,2 , and 4 provided feedback weekly over the study. Producer 3 provided weekly feedback from the start to September 2016. Producers may have been more willing to check alerts when the system was still novel. Decreased evaluations over time could have been linked to the novelty wearing off or to increased producer comfort with the system (knowing what was or was not linked to a disease event). Our results also suggested that producers failed to evaluate the alerts they deemed unimportant. Woodall and Montgomery (2014) suggested that too many alerts or alerts without a critical change would cause users to ignore alerts over time, like what we ob-

Table 9. Odds ratios and 95\% confidence intervals for system, ${ }^{1}$ day group, ${ }^{2}$ lactation stage,${ }^{3}$ heat stress, ${ }^{4}$ alerts per day, ${ }^{5}$ and behavior alerted ${ }^{6}$ effects on dairy producer cow alert ${ }^{7}$ evaluation when the cow alert was doubted and the cow was not visually checked based on the alert ${ }^{8}$

\begin{tabular}{|c|c|c|c|c|c|}
\hline Categorization $^{9}$ & Risk factor & Reference group & Odds ratio & $95 \% \mathrm{CI}^{10}$ & $P$-value \\
\hline \multicolumn{6}{|c|}{ OutsideInfluence vs. ChangeDoubted } \\
\hline & Version 2 & Version 1 & 14.67 & $6.36-33.83$ & $<0.01$ \\
\hline & Fresh lactation & Early lactation & 10.16 & $4.15-24.89$ & $<0.01$ \\
\hline & THI $<68$ & THI $\geq 68$ & 2.44 & $1.28-4.66$ & 0.01 \\
\hline & Combination & Eating & 7.88 & $4.06-15.31$ & $<0.01$ \\
\hline & Lying & Eating & 5.12 & $2.77-9.44$ & $<0.01$ \\
\hline OutsideInfluence vs. Other & Version 2 & Version 1 & 32.99 & $14.26-76.29$ & $<0.01$ \\
\hline & Weekend & Weekday & 1.67 & $1.14-2.43$ & $<0.01$ \\
\hline & Fresh lactation & Early lactation & 22.60 & $8.95-57.05$ & $<0.01$ \\
\hline & Fresh lactation & Post-peak lactation & 28.46 & $12.73-63.62$ & $<0.01$ \\
\hline & $\leq 20$ alerts $/ \mathrm{d}$ & $>20$ alerts $/ \mathrm{d}$ & 2.01 & $1.28-3.16$ & $<0.01$ \\
\hline & Weekend & Weekday & 4.14 & $2.58-6.65$ & $<0.01$ \\
\hline & Fresh lactation & Early lactation & 3.22 & $1.29-8.08$ & $<0.01$ \\
\hline & Fresh lactation & Post-peak lactation & 5.92 & $2.30-15.21$ & $<0.01$ \\
\hline & $\leq 20$ alerts $/ \mathrm{d}$ & $>20$ alerts/d & 3.47 & $2.03-5.92$ & $<0.01$ \\
\hline & Combination & Lying & 3.20 & $1.28-7.99$ & $<0.01$ \\
\hline & Eating & Lying & 5.90 & $3.08-11.28$ & $<0.01$ \\
\hline
\end{tabular}

${ }^{1}$ Software version was grouped as version 1 (pre-May 2016) or version 2 (post-May 2016). The software was updated on May 11, 2016, to a visually different interface, and cows in estrus were not included on the health and management list.

${ }^{2}$ Day group was weekday (Monday to Friday) or weekend (Saturday to Sunday).

${ }^{3}$ Lactation stage was grouped as fresh ( $\leq 30$ DIM), early (31-99 DIM), or post-peak ( $\geq 100$ DIM) lactation.

${ }^{4}$ Heat stress was grouped as temperature humidity index $\geq 68$ or $<68$.

${ }^{5}$ Alerts per day were grouped as $\leq 20$ or $>20$ cow alerts on the list per day. Twenty cow alerts corresponded to the default number displayed on the health and management list.

${ }^{6}$ Behavior alerted referred to eating time, lying time, or activity (steps/d) and any 2- or 3-way combination of the behaviors that decreased below the predetermined threshold and triggered an alert to be created on the health and management list.

${ }^{7}$ Cow alerts were generated for a change in activity (steps/d), lying time $(\mathrm{h} / \mathrm{d})$, or eating time $(\mathrm{h} / \mathrm{d})$ collected by wearable precision dairy technologies.

${ }^{8}$ Parity group was first, second, and third or more lactation. Parity group was not a significant influencer of OutsideInfluence, ChangeDoubted, or Other $(P=0.29)$.

${ }^{9}$ Within the cow alert category AlertDoubted (cow alert was doubted and cow was not visually checked), ChangeDoubted indicated that the alert was not considered to represent a real behavior change. OutsideInfluence indicated that the behavioral change was attributed to a pen change, dry-off, veterinary or pregnancy check, or hoof trimming. Other indicated that the producers wrote in their own response; common responses included cow repeatedly on the list, weather changes, and the producer had no time to visually assess cows (Table 2; Figure 1).

${ }^{10}$ Confidence intervals overlapping the null value (odds ratio $=1$ ) were not included in the table. 
served in our study. Overall alert evaluation decreased, but evaluated alerts were more likely to be CowCheck (cow alert perceived to be true and cow was visually checked) or NoAction (cow alert was perceived to be true, but the cow was not visually checked because of the alert) instead of AlertDoubted (cow alert was doubted and cow was not visually checked).

Producers were more likely to attribute behavioral changes to outside influences (e.g., pen changes, dryoffs, estrus events, veterinary examinations) the longer the system was in place. It is likely that, over time, producers had increased understanding of the system and made logical connections with management effects. Including dairy producers in technology creation or alert-generation strategies could help decrease the number of unnecessary alerts created by a system (Huirne et al., 1997; Wathes et al., 2008). Incorporating PDM into management software could also improve alert generation (Borchers and Bewley, 2015). If producers were able to enter pen changes, dry-offs, or scheduled events (e.g., pregnancy checks, hoof trimmings), technology companies could modify governing algorithms or alert thresholds based on the additional information. Although producers can recognize that the changes in behavior occurred because of outside influences, potential disease alerts attributed to those changes could result in decreased producer confidence in disease alerts, as it did in the current study.

\section{Day Group}

Day group played a crucial role in evaluations. Producers were more likely to evaluate alerts, check alerts, and identify cows as sick during the work week. Producers had less labor available on the weekends (personal communication) and were more likely to devote time to family (personal communication) than during the work week. Conversely, Hogeveen et al. (2013) stated that 7 producers using automated milking systems in the Netherlands did not change how often they checked a mastitis alert list between weekends and weekdays. However, Hogeveen et al. (2013) administered a questionnaire to gather how producers used mastitis alerts, followed up with 5 site visits. Tracking changes in how the alerts were used over time could have provided results like those in our study. Showing only urgent or high-priority alerts (fresh cows, mastitis, or calving alerts) on weekends could improve producer use of disease alerts.

\section{Lactation Stage}

Producers were more likely to evaluate alerts on cows in the fresh period or early lactation than cows in later lactation. Cows are at the highest disease risk during the transition period, 3 wk pre- to 3 wk postparturition (LeBlanc, 2010). Borchers and Bewley (2015) suggested that producers were more likely to use measurements they were more familiar with (steps/d and milk weights). The same could be true for which cow alerts producers prioritized. Producers may have increased alert evaluation during the fresh period and early lactation because of increased concern during that stage of a cow's life.

An increased likelihood of coding alerts as ChangeOk in fresh cows instead of Other was also seen. When talking with the producers, an idea was proposed. The alerts monitored eating time, lying time, and steps per day, and the possibility existed for changes in one variable to affect another. The producers noted that fresh cows with lying time alerts (decreased lying time) could correspond to increases in eating time. Many alerts were coded ChangeOk because of this, with producers viewing this situation as a positive change indicative of cows improving postpartum (J. Kiser, R. Mattingly, F. Rowe, and S. Sidebottom; personal communication). Huzzey et al. (2005) noted that eating frequency increased postpartum (9.8 vs. $11.1 \pm 0.5$ meals/d pre- and postpartum, respectively; $P=0.09)$ and that standing time increased postpartum (12.3 vs. $13.4 \pm 0.3 \mathrm{~h} / \mathrm{d}$ pre- and postpartum, respectively; $P=0.02$ ).

One limitation of the current alert creation strategy was that increased behaviors did not create disease alerts. Theoretically, if a cow increased lying time, eating time and activity would decrease, creating an alert. However, the behavior volatility around calving could have made establishing a baseline difficult. Huzzey et al. (2005) reported inconsistency within and across herds pre- and postpartum for feeding and drinking behavior, with standing time increasing only at the time of calving. With the behavioral volatility around calving, potentially providing different lists for different stages of lactation could improve producer use of disease alerts. Setting higher thresholds (above the $\geq 30 \%$ decrease set in our study) for cows in later lactation could decrease the number of unused alerts and enable producers to more closely focus on high-risk cows.

\section{Alerts per Day}

Similarly, having $\leq 20$ alerts on the alert list per day improved the likelihood of producers evaluating alerts and visually checking alerts. A small number of alerts was more manageable to examine both on and off the computer screen. To view more than 20 alerts, producers had to either view multiple pages or alter the number of alerts viewed at a single time. Increasing the number of alerts that can be viewed at a single time could improve alert use. Hogeveen et al. (2013) stated 
that producers spent a maximum of 10 min looking at a mastitis alert list. However, Hogeveen's producers saw between 6 and 30 udder health alerts per day. Minimizing data noise from pen changes or scheduled events could help restrict alerts to health-specific behavioral changes and decrease the number of alerts per day and the time needed to evaluate alerts. Adopting these changes could encompass the findings of Woodall and Montgomery (2014), Wathes et al. (2008), and Russell and Bewley (2013) that producers desire actionable, individualized alerts instead of information overload.

Additionally, producers could have associated a high number of alerts with increased false positives (i.e., an alert was created and the cow was healthy). Many studies have emphasized the importance of limiting false positives and negatives (sensitivity and specificity) with behavior-generated alerts (Hogeveen et al., 2010; Fricke et al., 2014; Borchers et al., 2017). In a companion study, sensitivity ranged from $18 \pm 3$ to $29 \pm 4 \%$ for the eating, lying, and activity alerts generated compared against disease events (any disease event: $24 \pm$ $3 \%$; hyperketonemia: $29 \pm 4 \%$; hypocalcemia: $29 \pm 3 \%$; metritis: $18 \pm 3 \%$; E. Eckelkamp, unpublished data). The results of the current study and the companion study indicated the producers had good reason to not evaluate alert lists with $>20$ alerts/d.

\section{Behavior Alerted}

Behavioral changes that created alerts affected at least 1 category within all groups. One of the most informative results was that producers were more likely to evaluate eating or activity alerts instead of lying alerts. The high number of lying alerts $(\mathrm{n}=9,777)$ compared with activity alerts $(\mathrm{n}=1,590)$ further emphasizes the importance producers placed on activity alerts. Although lying time biologically is vital for dairy cow health (Munksgaard et al., 2005; Ito et al., 2009, 2010), producers did not consider decreased lying time to be as actionable as decreased eating time or activity. Borchers and Bewley (2015) noted that producers were more willing to adopt technologies providing familiar measurements, particularly activity and milk yield. Dairy cattle activity monitoring (steps/d) has been linked to estrus detection since the 1950s (Farris, 1954) with pedometers. Increased familiarity with activity could have influenced producers' preference for activity alerts. Huirne et al. (1997) and Borchers and Bewley (2015) suggested that technologies with proven performance on familiar measurements were more likely to be adopted by dairy producers. Over time, lying time could become more readily accepted by dairy producers.
Producers were also more likely to categorize cows as visually ill in combination with eating alerts. In a companion study, eating time was a major influencer for random forests, principal component analysis neural networks, and least discriminate analyses techniques identifying diseases from behavioral patterns (E. Eckelkamp, unpublished data). Edwards and Tozer (2004), Clement et al. (2013), and Stangaferro et al. (2016a,b,c) all reported detection of health disorders based on rumination and activity. Producers may have seen decreased activity and eating time as more actionable than decreased lying time. In the future, focusing on individual health behaviors or behavior combinations with strong links to diseases could improve alert usefulness and disease identification.

\section{Limitations and Further Discussion}

A technology must fit within the farm management style and fill a need before it can be considered a good investment (Yule and Eastwood, 2012). Although our study took place in the first year of adoption, producers showed a willingness to learn and to provide insight into how first-time users viewed technology-generated disease alerts. Although our sample size was limited to 4 producers, this study did capture differences in management and style inherent to producer use of PDM. Producers as end users will have different priorities, biases, and management styles. Because of this, continued research with and incorporation of producers into PDM development and deployment are critical. Technology is also constantly being improved and updated. At the time of publication, a new sensor (Nedap SmartTag Neck; Nedap Livestock Management) was released, providing rumination, eating, active, and inactive time. These changes may influence producer use of technology alerts.

Although our sample size was small, several critical areas could be defined. Our study showed that producers placed a higher priority on eating or activity alerts, in fresh or early-lactation cows, within the typical work week, and when $\leq 20$ alerts were on the alert list. Producers were more willing to evaluate high-risk cows (fresh or early lactation), especially when eating time and activity decreased. Borchers and Bewley (2015) suggested that producers more readily adopted familiar measurements. The same could be suggested for measurements on cows already being observed. Producers were likely willing to evaluate alerts because they already visually monitored fresh and earlylactation cows for eating and activity and were willing to believe that the system was accurately monitoring them as well. 
Limited labor availability and low dairy prices will likely continue to drive PDM adoption in a quest for improved efficiency. However, producers may continue to be resistant to adopting technologies unless benefits are obvious and the technology is easy to learn and use (Spahr, 1993). Along with balanced accuracy, considering usability, perceived usefulness, and perceived accuracy could improve producer adoption.

\section{CONCLUSIONS}

Precision dairy technology has many potential uses, but more work is needed to improve disease detection alerts. Although producers indicated that most of the alerts represented a real behavioral change (55\%) and only $8 \%$ of the alerts were doubted, $37 \%$ of alerts were not evaluated, and producers visually followed up on only $21 \%$ of the alerts. Behavioral disease alerts must be improved and correspond to an actionable change for producers to use them. Producers were more likely to use eating or activity alerts, alerts in fresh or earlylactation cows, during the work week, and when $\leq 20$ alerts were on the list. Incorporating herd management software, creating and managing alerts by lactation stage, and focusing on behaviors producers already find useful could improve the use of alerts in the future.

\section{ACKNOWLEDGMENTS}

The authors thank the cooperating Kentucky farmers who made this study possible. We also thank Alta Genetics (Watertown, WI) for their financial support of this study and Nedap (Groenlo, the Netherlands) for their technical support of this study. We also thank Eric Roemmele (University of Kentucky, Lexington) for his time and statistical expertise. Finally, we thank the students at the University of Kentucky Dairy Science Program for their assistance with laboratory analysis and data entry.

\section{REFERENCES}

Bar, D., and R. Solomon. 2010. Rumination collars: What can they tell us. Pages 214-215 in Proc. 1st North Am. Conf. Precision Dairy Management, Toronto, ON, Canada.

Berckmans, D. 2015. Smart farming for Europe: Value creation through precision livestock farming. Pages $25-36$ in Precision Livestock Farming Applications. M. Guarino and D. Berckmans, ed. Wageningen Academic Publishers, Wageningen, the Netherlands.

Bewley, J. M., M. R. Borchers, K. A. Dolecheck, A. R. Lee, A. E. Stone, and C. M. Truman. 2017. Precision dairy monitoring technology implementation opportunities and challenges. Pages 12511261 in Large Dairy Herd Management. 3rd ed. D. K. Beede, ed. American Dairy Science Association, Champaign, IL.

Borchers, M. R., and J. M. Bewley. 2015. An assessment of producer precision dairy farming technology use, prepurchase considerations, and usefulness. J. Dairy Sci. 98:4198-4205.
Borchers, M. R., Y. M. Chang, K. L. Proudfoot, B. A. Wadsworth, A. E. Stone, and J. M. Bewley. 2017. Machine-learning-based calving prediction from activity, lying, and ruminating behaviors in dairy cattle. J. Dairy Sci. 100:5664-5674.

Borchers, M. R., Y. M. Chang, I. C. Tsai, B. A. Wadsworth, and J. M. Bewley. 2016. A validation of technologies monitoring dairy cow feeding, ruminating, and lying behaviors. J. Dairy Sci. 99:74587466.

Clement, P., R. Guatteo, J.-M. Philipot, J.-M. Lamy, A. Chanvallon, G. Trou, and N. Bareille. 2013. Potential use of drops in rumination time for real-time detection of health disorders in dairy cows. Pages 147-148 in Proc. North Amer. Conf. Precision Dairy Management, Rochester, MN.

Dolecheck, K. A., W. J. Silvia, G. Heersche Jr., C. L. Wood, K. J. McQuerry, and J. M. Bewley. 2016. A comparison of timed artificial insemination and automated activity monitoring with hormone intervention in 3 commercial dairy herds. J. Dairy Sci. 99:1506-1514.

Eckelkamp, E. A. 2019. Invited review: Current state of wearable precision dairy technologies in disease detection. Appl. Anim. Sci. $35: 209-220$

Edwards, J. L., and P. Tozer. 2004. Using activity and milk yield as predictors of fresh cow disorders. J. Dairy Sci. 87:524-531.

Farris, E. J. 1954. Activity of dairy cows during estrus. J. Am. Vet. Med. Assoc. 125:117-120.

Fricke, P. M., P. Carvalho, J. Giordano, A. Valenza, G. Lopes, and M. Amundson. 2014. Expression and detection of estrus in dairy cows: The role of new technologies. Animal 8:134-143.

Hogeveen, H., K. J. Buma, and R. Jorritsma. 2013. Use and interpretation of mastitis alerts by farmers. Pages 313-319 in Proc. 6th European Conf. Precision Livestock Farming, Leuven, Belgium. Wageningen Academic Publishers, Wageningen, the Netherlands.

Hogeveen, H., C. Kamphuis, W. Steeneveld, and H. Mollenhorst. 2010 Sensors and clinical mastitis - The quest for the perfect alert. Sensors (Basel) 10:7991-8009.

Huirne, R. B., S. B. Harsh, and A. A. Dijkhuizen. 1997. Critical success factors and information needs on dairy farms: The farmer's opinion. Livest. Prod. Sci. 48:229-238.

Huzzey, J. M., M. A. G. Von Keyserlingk, and D. M. Weary. 2005 Changes in feeding, drinking, and standing behavior of dairy cows during the transition period. J. Dairy Sci. 88:2454-2461.

Itle, A. J., J. M. Huzzey, D. M. Weary, and M. A. G. Von Keyserlingk. 2015. Clinical ketosis and standing behavior in transition cows. J. Dairy Sci. 98:128-134.

Ito, K., M. A. G. von Keyserlingk, S. J. LeBlanc, and D. M. Weary. 2010. Lying behavior as an indicator of lameness in dairy cows. J. Dairy Sci. 93:3553-3560.

Ito, K., D. M. Weary, and M. A. G. von Keyserlingk. 2009. Lying behavior: Assessing within- and between-herd variation in free-stallhoused dairy cows. J. Dairy Sci. 92:4412-4420.

Jago, J., C. Eastwood, K. Kerrisk, and I. Yule. 2013. Precision dairy farming in Australasia: Adoption, risks and opportunities. Anim. Prod. Sci. 53:907-916.

Jawor, P. E., J. M. Huzzey, S. J. LeBlanc, and M. A. G. von Keyserlingk. 2012. Associations of subclinical hypocalcemia at calving with milk yield, and feeding, drinking, and standing behaviors around parturition in Holstein cows. J. Dairy Sci. 95:1240-1248.

LeBlanc, S. 2010. Monitoring metabolic health of dairy cattle in the transition period. J. Reprod. Dev. 56:S29-S35.

Liberati, P., and P. Zappavigna. 2009. Improving the automated monitoring of dairy cows by integrating various data acquisition systems. Comput. Electron. Agric. 68:62-67.

Liboreiro, D. N., K. S. Machado, P. R. B. Silva, M. M. Maturana, T. K. Nishimura, A. P. Brandão, M. I. Endres, and R. C. Chebel. 2015. Characterization of peripartum rumination and activity of cows diagnosed with metabolic and uterine diseases. J. Dairy Sci. 98:6812-6827.

Mazrier, H., S. Tal, E. Aizinbud, and U. Bargai. 2006. A field investigation of the use of the pedometer for the early detection of lameness in cattle. Can. Vet. J. 47:883-886. 
Miekley, B., I. Traulsen, and J. Krieter. 2012. Detection of mastitis and lameness in dairy cows using wavelet analysis. Livest. Sci. 148:227-236.

Munksgaard, L., M. B. Jensen, L. J. Pedersen, S. W. Hansen, and L. Matthews. 2005. Quantifying behavioural priorities - Effects of time constraints on behaviour of dairy cows, Bos taurus. Appl. Anim. Behav. Sci. 92:3-14.

NOAA (National Oceanic and Atmospheric Administration). 1976. Livestock hot weather stress. Operations Manual Lett. C-31-76. NOAA, Silver Spring, MD.

Russell, R. A., and J. M. Bewley. 2013. Characterization of Kentucky dairy producer decision-making behavior. J. Dairy Sci. 96:47514758 .

Rutten, C. J., A. Velthuis, W. Steeneveld, and H. Hogeveen. 2013. Invited review: Sensors to support health management on dairy farms. J. Dairy Sci. 96:1928-1952.

Spahr, S. L. 1993. New technologies and decision making in high producing herds. J. Dairy Sci. 76:3269-3277.

Stangaferro, M. L., R. Wijma, L. S. Caixeta, M. A. Al-Abri, and J. O. Giordano. 2016a. Use of rumination and activity monitoring for the identification of dairy cows with health disorders: Part I. Metabolic and digestive disorders. J. Dairy Sci. 99:7395-7410.

Stangaferro, M. L., R. Wijma, L. S. Caixeta, M. A. Al-Abri, and J. O. Giordano. 2016b. Use of rumination and activity monitoring for the identification of dairy cows with health disorders: Part II Mastitis. J. Dairy Sci. 99:7411-7421.
Stangaferro, M. L., R. Wijma, L. S. Caixeta, M. A. Al-Abri, and J. O. Giordano. 2016c. Use of rumination and activity monitoring for the identification of dairy cows with health disorders: Part III. Metritis. J. Dairy Sci. 99:7422-7433.

Van Erp-Van der Kooj, E., M. Van de Brug, and J. B. Roelofs. 2016. Validation of Nedap Smarttag leg and neck to assess behavioural activity level in dairy cattle. Pages $321-326$ in Proc. Precision Dairy Farming 2016. Wageningen Academic Publishers, Wageningen, the Netherlands.

Wathes, C. M., H. H. Kristensen, J. M. Aerts, and D. Berckmans. 2008. Is precision livestock farming an engineer's daydream or nightmare, an animal's friend or foe, and a farmer's panacea or pitfall? Comput. Electron. Agric. 64:2-10.

Woodall, W. H., and D. C. Montgomery. 2014. Some current directions in the theory and application of statistical process monitoring. J. Qual. Technol. 46:78-94.

Yule, I., and C. Eastwood. 2012. Challenges and opportunities for precision dairy farming in New Zealand. Pages 15-18 in Proc. 11th Int. Conf. Precision Ag., Indianapolis, IN. J. Stafford, ed. https:// www.ispag.org/abstract_papers/papers/abstract_1264.pdf.

\section{ORCIDS}

E. A. Eckelkamp (1) https://orcid.org/0000-0003-0141-7839

J. M. Bewley ๑ https://orcid.org/0000-0002-7347-2741 